\title{
Skin Doses on the Lens for Temporomandibular Joint Exam in Cone Beam Computed Tomography
}

\author{
Marcus Vinicius Linhares de Oliveira ${ }^{1,3^{*}}$ Marcos Ely Almeida Andrade $^{2}$, Wilson Otto \\ Batista $^{3}$ and Paulo Sergio Flores Campos ${ }^{4}$ \\ ${ }^{1}$ Instituto de Ciências da Saúde; Universidade Federal da Bahia; Salvador - BA - Brasil. ${ }^{2}$ Departamento de Energia \\ Nuclear; Centro de Tecnologia; Universidade Federal de Pernambuco; Recife - PE - Brasil. ${ }^{3}$ Departamento de \\ Tecnologia em Saúde e Biologia; Instituto Federal da Bahia; Salvador - BA - Brasil. ${ }^{4}$ Departamento de Radiologia \\ Oral; Faculdade de Odontologia; Universidade Federal da Bahia; Salvador - BA - Brasil
}

\begin{abstract}
The aim of this study was to evaluate the kerma at the surface of the lens in TMJ CBCT and to derive the equivalent dose. An anthropomorphic phantom of the head and neck manufactured by Radiation Support Devices (model RS230) was used. The dosimetric measurements were obtained by using fourteen thermoluminescent dosimetry (TLD) dosimeters ( $\mathrm{LiF}: \mathrm{Mg}, \mathrm{Ti}$ ), divided in two pairs (one pair for each eye) and positioned on the surface of the phantom, per scanner evaluated. The tomographic images were acquired in three types of CBCT equipment (CS 9000, Gendex GXCB 500 and $i$-CAT). Values of equivalent dose obtained were: $5.82 \mathrm{mSv}$ (CS 9000); $5.38 \mathrm{mSv}$ (Gendex GXCB $500)$ and $7.98 \mathrm{mSv}$ (i-CAT), which varied in accordance with the scanner and the exposure factors used in the image acquisition. The Gendex GXCB 500 used larger FOV and higher $k V$, resulting in levels close to those obtained on the CS 9000, while larger doses were associated with the i-CAT. The dose values associated with TMJ radiological procedures should be performed with awareness and appropriateness due to sensitivity of the lens.
\end{abstract}

Key words: cone beam computed tomography, temporomandibular joint, radiation dosimetry

\section{INTRODUCTION}

The temporomandibular joint (TMJ) is a synovial joint formed by the mandibular condyle, glenoid fossa and the articular eminence of the temporal bone. It is considered one of the more complex structures of the human body and supports chewing and speaking functions (Gedrange et al. 2012). The changes that occur in the auxiliary and muscle structures of this joint affect $33 \%$ of the population (Palconet et al. 2012) Traditionally, there are several imaging methods that are used to assess the changes in bone components of the joint, such as panoramic radiography, conventional radiography and computed tomography, while magnetic resonance imaging (MRI) is useful in the evaluation of soft tissue (Barghan et al. 2012). For the detection of bone changes, panoramic radiography, for example, is unreliable, unlike cone beam computed tomography (CBCT), which offers great advantages for viewing osseous components in the three planes of space, presents excellent visualization of bone changes and is a useful method in evaluating craniofacial structures, including the temporomandibular joint (TMJ) ( Librizzi et al. 2011)

The radiological protection guide for CBCT developed by the Safety and Efficacy of a New Emerging Dental X- Ray project (Sedentexct

*Author for correspondence: marcusradiology@gmail.com 
2011) aims to establish care that justifies the rational use of dental and maxillofacial radiology, and presents criteria for possible diagnoses and imaging methods available. Thus, knowing the radiation dose values that patients who undergo CBCT scans are being subjected to is crucial, especially because of the lack of uniformity in the configuration of CBCT equipment with different voltages, variable fields of view (FOV) and devices that provide 180,210 and $360^{\circ}$ rotations (Batista et al. 2013). The European Guidelines on Radiation Protection in Dental Radiology (European Commission, 2014) recommends the quality of the image and the reference doses in dental radiology, but this guide does not mention the CBCT. Some studies have shown ways to reduce the radiation dose in $\mathrm{CBCT}$, such as reducing the size of the FOV or using lower $\mathrm{kV}$ and mAs (Ludlow et al. 2006; Palomo et al. 2008; Qu et al. 2010).

Currently, the international committee has revised the radiation dose limits for certain radiationsensitive organs, such as the lens. The limit for the lens was $150 \mathrm{mSv}$ dose equivalent per year and it was reviewed by ICRP in 2011. The new value of $20 \mathrm{mSv}$ was established as an average over five years, indicating that this structure is more delicate than previously thought. There has been a noted increase in performing this type of examination without an established diagnostic reference level (DRL). Thus, the purpose of this study was to evaluate the kerma on the lens in examining the TMJ in CBCT and to derive the equivalent dose.

\section{MATERIAL AND METHODS}

This study was conducted by acquiring TMJ images from an anthropomorphic phantom of the head and neck (Radiation Support Devices, model RS-230), with the same density and atomic number of bone and soft tissue of an average adult male patient that reproduced the same density of an average sized patient. The images were obtained in three types of CBCT equipment: CS 9000 (Carestream Health, New York, New York); Gendex GXCB 500 (Gendex Dental Systems, Pennsylvania, USA) and i-CAT classic (Imaging International Sciences, Hatfield, Pennsylvania, USA), (Fig. 1).
(A)

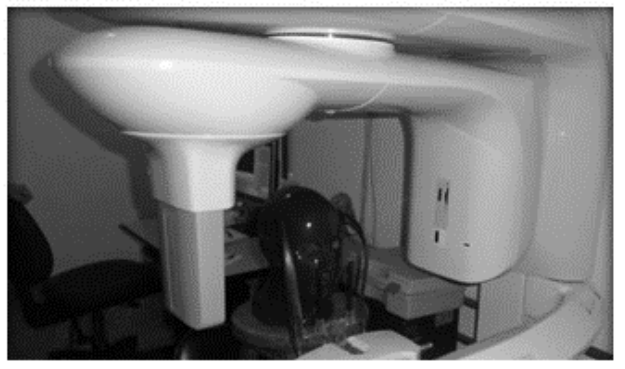

(B)

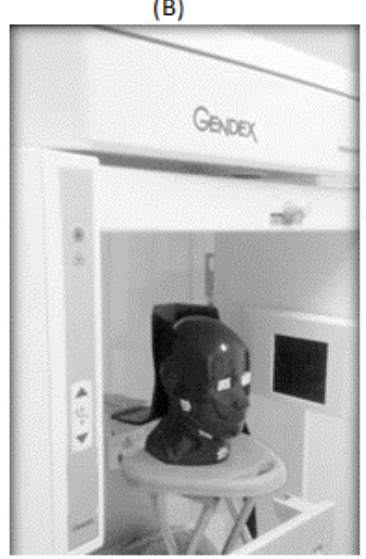

(C)

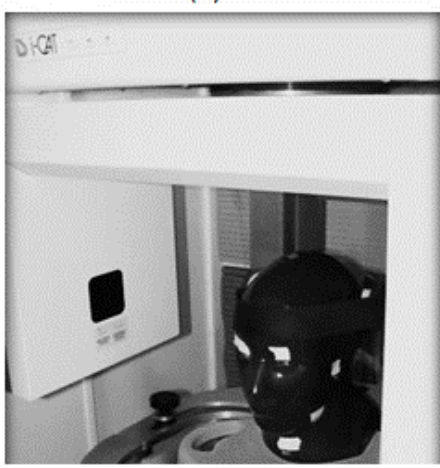

Figure 1 - Equipment of CBCT: A) CS 9000 3D, B) Gendex CB 500 and C) i-CAT classic.

The images were obtained with the TMJ standard protocols defined by the manufacturer. For the CS 9000, as it was a device with small limited FOV (50 $\mathrm{mm} \times 37 \mathrm{~mm}$ ), only one TMJ was obtained by exposure. The joint was centralized through the position of the laser light projected on the TMJ studied. The leadmarks was placed horizontally at $3.5 \mathrm{~cm}$ above the external acoustic meatus and vertically at $1.0 \mathrm{~cm}$ ahead of the same structure.
Table 1 presents the protocols performed in the three types of equipment. For the Gendex GXCB 500 and i-CAT, two joints were included in one exposure. A routine TMJ evaluation was done by the exposure with an open mouth and closed mouth. Therefore, two exhibitions exposures were made in i-CAT and Gendex GXCB 500 and four for CS 9000. The joint was centralized with the support of a positioning laser point. The 
anthropomorphic phantom was centered with the mid-sagittal plan over the isocenter of the CT scanner. Seven pairs of thermoluminescent dosimeters (TLD) (LiF: Mg, Ti) positioned on the surface of the lens and divided into two pairs (one for each eye) per scanner were used. One pair of dosimeters was used to evaluate the background radiation and was, therefore, not exposed to the $\mathrm{x}$ ray beam from the CBCT scanner, and was sent to the reader with other exposed dosimeters. The absorbed dose determined for each TLD was the result of the dose response curve, obtained by considering the correction factor related to the calibration and reference light at the time of reading. The coefficient of variation of TLD was $8 \%$ and maximum uncertainty was estimated in $10 \%$.

Table 1 - Exposure protocols on CBCT for TMJ.

\begin{tabular}{ccccccc}
\hline $\begin{array}{c}\text { CBCT } \\
\text { Equipment }\end{array}$ & $\mathbf{k V p}$ & $\begin{array}{c}\text { HVL } \\
\text { mAs }\end{array}$ & $\begin{array}{c}\text { [mm } \\
\text { Al] }\end{array}$ & $\begin{array}{c}\text { Voxel } \\
{[\mathbf{m m}]}\end{array}$ & $\begin{array}{c}\text { FOV } \\
{[\mathbf{d} \times \mathbf{h}} \\
\mathbf{m m}]\end{array}$ & $\begin{array}{c}\text { X ray } \\
\text { source - } \\
\text { detector } \\
\text { distance } \\
\text { [cm] }\end{array}$ \\
\hline CS 9000 & 70 & 108 & 2.6 & 0.076 & $50 \times 37$ & 54 \\
i-CAT & 120 & 36 & 9.4 & 0.250 & $160 \times 80$ & 68 \\
Gendex & 120 & 30 & 9.3 & 0.250 & $140 \times 80$ & 68 \\
GXB500 & & & & & & \\
\hline
\end{tabular}

The dosimeters were calibrated according to the photons energy levels emitted by the CBCT scanner. After the exposure, the TLDs were sent to the reader Victoreen 2800. The TLD's were exposed twice assuring greater reliability of dose readings in the protocols used and consequently divided by two. With this data, the equivalent dose was estimated using the following equation:

$H_{T}=D_{a b s} \times w_{R} ; D_{a b s} \approx$ Kerma

where $\mathrm{H}_{T}$ was the equivalent dose (mSv), $\mathrm{D}_{\mathrm{abs}}$ was the absorbed dose and $\mathrm{W}_{\mathrm{R}}$ was a factor of weighting of radiation, once it was related to $\mathrm{X}$ rays it was equal 1 .

\section{RESULTS AND DISCUSSION}

From the three types of equipment, the CS 9000 stood out because it used exposure protocols with lower voltages $(70 \mathrm{kV})$ and high current time product ( 108 mAs), while the Gendex GXCB 500 and i-CAT operate only with $120 \mathrm{kV}$ with $30 \mathrm{mAs}$ and $36 \mathrm{mAs}$, respectively. The kerma on the surface of the eye for the right and left side is shown in Table 2. The i-CAT presented higher doses (approximately 30\%) compared to the other two equipment types. Oliveira et al. 2014, showed these results previously. Even the CS 9000, which worked with lower voltages $(70 \mathrm{kV})$, had the same dose as the Gendex GXCB 500 using $120 \mathrm{kV}$.

Radiation doses on the lenses and its evaluation are essential. Recently, the International Commission on Radiological Protection (ICRP) reviewed the epidemiological evidence suggesting that the dose value for cataracts should be below $0.5 \mathrm{~Gy}$, instead of $2.0 \mathrm{~Gy}$ and the occupational exposure limit changed to $20 \mathrm{mSv}$ per year (ICRP 2011), suggesting that the lens of the eye could be more radiosensitive than previously considered. This demonstrated the need to improve the protocols that aimed to reduce the risks for this organ and improved the care that must be taken with exposure repetition on the same patient, mainly due to poor positioning of the patient. Another relevant point was to evaluate the absolute necessity in performing a cone beam computed tomography exam by considering other diagnosis options.

Table 2 - Doses on lens of three types of CBCT equipment.

\begin{tabular}{lcccc}
\hline $\begin{array}{c}\text { CBCT } \\
\text { Equipment }\end{array}$ & \multicolumn{3}{c}{$\begin{array}{c}\text { Surface Kerma on } \\
\text { lens [mGy] }\end{array}$} & $\begin{array}{c}\text { Equivalent } \\
\text { Doses [mSv] }\end{array}$ \\
\hline & right & left & average & \\
CS 9000 & 5.2 & 6.5 & 5.8 & 5.8 \\
i-CAT & 6.8 & 9.1 & 7.9 & 7.9 \\
Gendex GXB500 & 4.9 & 5.9 & 5.4 & 5.4 \\
\hline
\end{tabular}

CT and MRI have often been used when studying TMJ, the latter allowing for the evaluation of bone components and soft tissue without exposure to ionizing radiation. There are, however, setbacks such as long scanning times and the restricted use due to its high cost (Tsiklakis et al. 2004). Despite the CBCT having lower radiation doses, it is imperative to know the dose levels in different equipment and operating modes, and to implement strategies to reduce doses due to the risks that arise from cumulative x-ray use (Ludlow and Ivanovic 2008).

A discrepancy in the skin doses for the scanners evaluated were noted. This was also noticeable in the work of Ludlow and Ivanovic (2008). The iCAT classic presented higher doses for the lens compared to the other two scanners. Even using high voltage and FOV, the Gendex GXCB 500 
presented similar doses to the CS9000. Thus, despite the CS 9000 having limited FOV, it did not result in reduced doses. In this case, other factors were associated with increased doses, such as the use of a low voltage and short focus- detector distance, due the low-energy photons to be easily attenuated on the surface of the organ. Also, the increase in the number of repetitions by positioning error became more evident due to the fact that the CS 9000 had smaller and limited FOV, making the correct positioning of the centralized structure with the isocenter more difficult. It would be worth to mention the need to constantly train professionals who perform the procedures so that repetitions of examinations caused by positioning error could be avoided.

The CT scanners Gendex and i-CAT have similar characteristics in terms of the voltage, tubedetector distance and filter used; however, when examining the results obtained, the influence of the current time product on the dose for the lens could be seen, which in the i-CAT was $20 \%$ higher. However, the difference in equivalent dose values between the two devices was $48 \%$. Another factor related to the lower doses obtained in the Gendex was the use of an asymmetrical beam, as described by Scarfe and Farman (2008) and Batista et al. (2015). This asymmetric beam was obtained by decentralizing the isocenter of the beam so that the central beam focused on the object's margin, focusing on one side more than the other. With this geometric maneuver, it was possible to increase the diameter of the image without changing the aperture of the collimator reducing dose levels in the lens region. This, together with the mAs, explained the resulting difference between the two devices.

Schulze et al. (2004) using CBCT equipment NewTom 9000, acquired images from an anthropomorphic phantom head and neck (similar to this study), in which TLDs were inserted to evaluate the dosimetric measurements, with a lens dose of $4.6 \mathrm{mGy}$. This result was very close to the present result. However, it was only one exposure of the maxilla and mandible. Palomo et al. (2008) evaluated the influence of exposure conditions on radiation doses on a MercuRay CBCT scanner and found out that using the protocol for $15 \mathrm{mAs}, 120$ $\mathrm{kVp}$ and FOV of 12 inches $(-30.48 \mathrm{~cm})$, the dose for the lens was $11.3 \mathrm{mGy}$. In another study using the same equipment, $16.5 \mathrm{mGy}$ for the lens was obtained (Ludlow et al. 2006). This was because MercuRay used FOV's that were considered large, while in the present study, the maximum FOV was $140 \mathrm{~mm} \times 85 \mathrm{~mm}$.

Two previous studies showed the use of lead glasses as a way of significantly reducing the doses in the lenses, reducing the risk of cataracts. The lead glasses could be useful because they were inexpensive and could be used in both the procedures with limited to total face FOV's (Prins et al. 2011; Goren et al. 2013). Considering TMJ image acquisition, the lead glasses become a valuable tool in reducing the radiation doses because the joint lies in the same horizontal plane of the lens, allowing the structure to be within the primary beam.

\section{CONCLUSION}

The equivalent doses obtained in this study were considered low and dependent of each CBCT scanner. The equivalent doses in the lens were not reduced when using the equipment with limited FOV. The dose values associated with TMJ radiological procedures should be performed with awareness and appropriateness due to sensitivity of the lens.

\section{ACKNOWLEDGEMENTS}

The authors thank the Laboratory of Physical Radiology of the Federal Institute of Bahia (LAFIR/IFBA), the Laboratory of Metrology of Ionizing Radiations of the Federal University of Pernambuco (LMRI-DEN/UFPE), for kindly allowing the use of the instruments used in the dosimetric evaluation by making available the instruments for the dosimetric evaluation, and the Lab TCFC 3D (Laboratory of Cone Beam Computed Tomography) of the Dental Faculty of the Federal University of Bahia, for their support in conducting this study.

\section{REFERENCES}

Barghan S, Tetradis S, Mallya S. Application of cone beam computed tomography for assessment of the temporomandibular joints. Aust Dent J. 2012; 57(Suppl. 1):109-118.

Batista WO, Navarro MV, Maia AF. Development of a phantom and a methodology for evaluation of depth Kerma and Kerma Index for dental Cone Beam Computed Tomography. Radiat Prot Dosimetry. 2013; 157(4): 543-551. 
Batista WO, Soares MR, de Oliveira MV, Maia AF, Caldas LV. Assessment of protocols in cone-beam CT with symmetric and asymmetric beams using effective dose and air kerma-area product. Appl Radiat Isto. 2015. doi:10.1016/ j.apradiso.2015.01.014.

European Commission. Radiation protection 136. European guidelines on radiation protection in dental radiology: Office for Official Publications of the European Communities. 2004.

Gedrange T, Gredes T, Hietschold V, Kunert-Keil $\mathrm{C}$, Dominiak M, Gerber $\mathrm{H}$, et al. Comparison of reference points in different methods of temporomandibular joint imaging. Adv Med Sci. 2012;57(1):157-162.

Goren A, Prins RD, Dauer LT, Quinn B, Al-Najjar A, Faber RD, et al. Effect of leaded glasses and thyroid shielding on cone beam CT radiation dose in an adult female phantom. Dentomaxillofac Radiol. 2013;42(6); 1-7.

ICRP . International Commission on Radiological Protection. Statement on Tissue Reactions, Seoul, Korea. 2011; 1-2.

Librizzi ZT, Tadinada AS, Valiyaparambil JV, Lurie AG, Mallaya SM. Cone-beam computed tomography to detect erosions of the temporomandibular joint: effect of field of view and voxel size on diagnostic efficacy and effective dose. Am J Orthod Dentofacial Orthop. 2011;140(1); 25-30.

Ludlow JB, Davies-Ludlow LE, Brooks SL, Howerton WB. Dosimetry of $3 \mathrm{CBCT}$ devices for oral and maxillofacial: CB Mercuray, NewTom 3G and iCAT. Dentomaxillofac Radiol. 2006; 35:219-226.

Ludlow JB, Ivanovic M. Comparative dosimetry of dental CBCT devices and 64-slice CT. Oral Surg Oral Med Oral Pathol Oral Radiol Endod. 2008;106(1); 106-114

Oliveira, MVL, Andrade, MEA, Soares, MR, Batista, WOG, Campos, PSF. Assessment of equivalent dose on the lens in cone beam computed tomography. in: xiv International Symposium On Solid State Dosimetry, ISSD 2014, 2014. p. 875-882.
Palconet G, Ludlow JB, Tyndall DA, Lim PF. Correlating Cone Beam CT Results with Temporomandibular joint pain of Osteoarthritic origin. Dentomaxillofac Radiol. 2012;41(2);126-130.

Palomo JM, Rao SP, Hans MG. Influence of CBCT exposure conditions on radiation dose. Oral Surg Oral Med Oral Pathol Oral Radiol Endod. 2008; 105 (6); 773-782.

Prins R, Dauer LT, Colosi DC, Quinn B, Kleiman NJ, Bohle GC, et al. Significant reduction in dental cone beam computed tomography (CBCT)eye dose through the use of leaded glasses. Oral Surg Oral Med Oral Pathol Oral Radiol Endod. 2011; 112(4); 502-507.

Qu XM, Li G, Ludlow JB, Zhang ZY, Ma XC. Effective radiation dose of ProMax 3D cone-beam computerized tomography scanner with different dental protocols. Oral Surg Oral Med Oral Pathol Oral Radiol Endod. 2010: 110(6); 770-776.L.

Scarfe WC, Farman AG. What is a cone beam CT and how does it work? Dental Clinics. 2008;52 (4): 707730 .

Schulze D, Heiland M, Thurmann H, Adam G. Radiation exposure during midfacial imaging using 4and 16-slice computed tomography, cone beam computed tomography systems and conventional radiography. Dentomaxillofac Radiol. 2004; 33(2);83-86.

Sedentexct Project. Radiation protection: cone beam CT for dental and maxillofacial radiology. Evidence based guideline. 2011, Available from: "http://www.sedentexct.eu/system/files/sedentexct_pr oject_provisional_guidelines.pdf.

Tsiklakis K, Syriopoulos K, Stamatakis HC. Radiographic examination ofthe temporomandibular joint using cone beam computed tomography. Dentomaxillofac Radiol. 2004; 33, (3):196-201. 